\title{
RELAÇÃO DE PARECERISTAS 2011
}

A Revista Estudos Interdisciplinares sobre o Envelhecimento agradece aos consultores, abaixo relacionados, que durante o ano de 2011 contribuíram analisando textos submetidos. Muito Obrigado!

Alarcon Agra Do Ó - Universidade Federal de Campina Grande

Ângelo José Gonçalves Bós - Pontifícia Universidade Católica do Rio Grande do Sul

Andrea Cristina Garofe Fortes Burgos - Universidade Federal de São Carlos

Alessandra Conceição Leite Fuchal Camacho - Universidade Estácio de Sá

Amer Cavalheiro Hamdan - Universidade Federal do Paraná

Anna Cruz de Araújo Pereira da Silva - Universidade Federal do Pará

Ana Maria Domingos - Universidade Federal do Rio de Janeiro

Anelise Coelho Fonseca - Escola Nacional de Saúde Pública

Andréa Krüger Gonçalves - Universidade Federal do Rio Grande do Sul

Álvaro Campos Cavalcanti Maciel - Universidade Federal do Rio Grande do Norte

Alessandra Samelli - Universidade de São Paulo

Ana Paula Santana - Universidade Federal de Santa Catarina

Adriana Cardoso de Oliveira Silva - Universidade Federal Fluminense

Alba Benemérita Alves Vilela - Universidade Estadual do Sudoeste da Bahia

Anderson Saranz Zago - Universidade Estadual Paulista Júlio de Mesquita Filho

Beatriz Aparecida Ozello Gutierrez - Universidade de São Paulo

Bruniele Olmo - Fisioterapeuta Rio de Janeiro

Beatriz Pinto Venancio - Universidade Federal Fluminense

Cláudia Burlá - Sociedade Brasileira de Geriatria e Gerontologia

Célia Pereira Caldas - Universidade do Estado do Rio de Janeiro

Cornelia Eckert - Universidade Federal do Rio Grande do Sul

Cíntia De La Rocha Freitas - Universidade Federal de Santa Catarina

Clezio José dos Santos Gonçalves - Universidade Federal do Rio Grande do Sul

Cristine Koehler Zanella - Faculdade de Direito de Santa Maria

Clarice Ehlers Peixoto - Universidade do Estado do Rio de Janeiro 


\section{Sul \\ Carlos Alberto Porcino - Especialização pela Universidade Cruzeiro do}

Claus Dieter Stobäus - Pontifícia Universidade Católica do Rio Grande
do Sul

Clarissa Marceli Trentini - Universidade Federal do Rio Grande do Sul

Carla Witter - Universidade São Judas Tadeu

Denise Ren da Fontoura - Universidade Federal do Rio Grande do Sul

Deborah Viviane Ferrari - Universidade de São Paulo

Dênis Marcelo Modeneze - Universidade Estadual de Campinas

Edmundo de Drummond Alves Junior - Universidade Federal Fluminense

Erika Barioni Mantello - Universidade de São Paulo

Eliane Jost Blessmann - Universidade Federal do Rio Grande do Sul

Eliane Pinheiro de Morais - Universidade Federal do Rio Grande do Sul

Erika Silva Maciel - Universidade de São Paulo

Eulália Maria Chaves Maia - Universidade Federal do Rio Grande do Norte

Edna Martins - Universidade Federal de São Paulo

Everton Massaia - Universidade Feevale

Eliane Cristina Hilberath Moreira - Universidade Estadual de Londrina

Eliete Jussara Nogueira - Universidade de Sorocaba

Eveline Torres Pereira - Universidade Federal de Viçosa

Eliane Ramos Pereira - Universidade Federal Fluminense

Fernando Berriel - Universidad de la Republica Uruguaya - Uruguay

Fátima Branco-Barreiro - Universidade Bandeirante de São Paulo

Fátima Helena Espírito Santo - Universidade Federal Fluminense

Gislaine Bonardi - Hospital Moinhos de Vento - RS

Helena Brandão Viana - Centro Universitário Adventista de São Paulo

Henrique Salmazo da Silva - Universidade de São Paulo

Helena Akemi Wada Watanabe - Universidade de São Paulo

Inês Henningen - Universidade Federal do Rio Grande do Sul

Iaci Proença Palmeira - Universidade do Estado do Pará

Ivalina Porto - Universidade Federal de Rio Grande

Janine Gomes Cassiano - Universidade Federal de Minas Gerais

Joseane Rodrigues da Silva Nobre - Universidade Estadual do Oeste do Paraná

Johannes Doll - Universidade Federal do Rio Grande do Sul

José Francisco Pinto de Almeida Oliveira - Sociedade Brasileira de Geriatria e Gerontologia - RJ 
José Sterza Justo - Universidade Estadual de São Paulo

Julio César Moriguti - Universidade de São Paulo

Joelma Batista Tebaldi Pinto - Universidade Estadual de Santa Cruz BA

Josiane Siviero - Universidade de Caxias do Sul

Jaqueline Girnos Sonati - Universidade Estadual de Campinas

Kayla Araújo Ximenes Aguiar Palma - Universidade Federal de Santa Maria

Lisianne Brittes Benitez - Universidade de Santa Cruz do Sul

Luciana Branco da Motta - Universidade do Estado do Rio de Janeiro

Lenisa Brandão - Universidade Federal do Rio Grande do Sul

Lucia Hisako Takase Gonçalves - Universidade Federal de Santa Catarina

Lilia Braga Maia - Universidade de Fortaleza

Leandro Fernandes Malloy-Diniz - Universidade Federal de Minas Gerais

Luis Paulo Gomes Mascarenhas - Universidade Federal do Paraná

Lucia Figueiredo Mourão - Universidade Estadual de Campinas

Larissa Chaves Pedreira - Universidade Federal da Bahia

Ligia Py - Universidade Federal do Rio de Janeiro

Lilian Ramos Sampaio - Universidade Federal da Bahia

Luís Henrique Telles Da Rosa - Universidade Federal de Ciências da Saúde de Porto Alegre

Leonéa Vitoria Santiago - Universidade Federal de Alagoas

Maria Helena Morgani de Almeida - Universidade de São Paulo

Mára Lúcia Fernandes Carneiro - Universidade Federal do Rio Grande do Sul

Maria Cristina Chimelo Paim - Universidade Luterana do Brasil

Márcia Regina Cubas - Pontifícia Universidade Católica do Paraná

Maria Josefina da Silva - Universidade Federal do Ceará

María Isolina Dabove - Universidad Nacional do Rosário - Argentina

Mara Villas Boas de Carvalho - Centro Universitário da Fundação de Ensino Octávio Bastos

Marcelo Filgueiras - Universidade Federal do Piauí

Maria Cristina França - Instituto Federal de Educação, Ciência e Tecnologia do Rio Grande do Sul

Mariana Cardoso Guedes - Centro Universitário São Camilo - SP

Maria das Graças Sobreira Leal - Instituto Sedes Sapientia

Marluce Auxiliadora Borges Glaus Leão - Universidade de Taubaté 
Maria Helena Lenardt - Universidade Federal do Paraná

Maria Emília Limeira Lopes - Universidade Federal da Paraíba

Marta Julia Marques Lopes - Universidade Federal do Rio Grande do Sul

Maria Do Rosário Menezes - Universidade Federal da Bahia

Myrian Spinola Najas - Universidade Federal de São Paulo

Maria Tereza de Souza Neves - Faculdade de Formação de Professores de Goiana - PE

Maria de Fátima Nunes Marucci - Universidade de São Paulo

Maira Rozenfeld Olchik - Universidade Federal do Rio Grande do Sul

Mônica de Oliveira Orsi Gameiro - Universidade Estadual Paulista Júlio de Mesquita Filho

Marlene Teda Pelzer - Universidade Federal de Rio Grande

Marilene Rodrigues Portella - Universidade de Passo Fundo

Marineia Crosara de Resende - Universidade Federal de Uberlândia

Mariana Tirolli Rett - Universidade Federal de Sergipe

Minéia Carvalho Rodrigues - Universidade Federal do Mato Grosso

Marisete Peralta Safons - Universidade de Brasília

Marcos Eduardo Scheicher - Universidade Estadual de São Paulo

Mauro Silveira - Universidade Federal do Rio Grande do Sul

Marina Borges Teixeira - Universidade de Guarulhos

Maria Helena Cirne De Toledo - Faculdades Associadas de Ensino - Boa Vista

Marilia Velardi - Universidade de São Paulo

Mônica Sanches Yassuda - Universidade de São Paulo

Neila Osório - Universidade Federal do Tocantins

Odair Perugini de Castro - Universidade Federal do Rio Grande do Sul

Paula Costa Castro - Universidade Federal de São Carlos

Pierre Normando Gomes da Silva - Universidade Federal da Paraíba

Patrícia Peres Oliveira - Universidade Paulista

Pericles Saremba Vieira - Universidade de Passo Fundo

Paulo José Zimermann Teixeira - Universidade Federal de Ciências da Saúde de Porto Alegre

Rochele Paz Fonseca - Pontifícia Universidade Católica do Rio Grande do Sul

Robert Pérez Fernández - Universidad de la República - Uruguay

Renata Coelho Scharlach - Universidade Federal de São Paulo

Sandra Marcia Ribeiro Lins de Albuquerque - Universidade de São Paulo 
Sheila Andreoli Balen - Universidade Federal do Rio Grande do Norte Silvia Luci de Almeida Dias - Universidade Federal do Pampa Sueli Aparecida Freire - Universidade Federal de Uberlândia Silvana Frota - Universidade Federal do Rio de Janeiro

Sebastião Gobbi - Universidade Federal de São Paulo

Sonia Lima Medeiros - Instituto Dante Pazzanese de Cardiologia - SP

Sofia Cristina Iost Pavarini - Universidade Federal de São Carlos

Sandra Vieira Larratéa - Universidade Federal do Rio Grande do Sul

Tomiko Born - Sociedade Brasileira de Geriatria e Gerontologia

Tereza Rosa Lins Vieira - Universidade Federal do Ceará

Tânia Maria De Oliva Menezes - Universidade Federal da Bahia

Verônica Bohm - Faculdade da Serra Gaúcha

Vitória Kachar Hernandes - Universidade São Judas Tadeu

Vagner Reolon Marcelino - Faculdade de Americana

Vera Maria Antonieta Tordino Brandão - Pontifícia Universidade Católica de São Paulo

Wildoberto Batista Gurgel - Universidade Federal do Maranhão 